\title{
Interactive comment on "Seasonal characteristics of emission, distribution and radiative effect of marine organic aerosols over the western Pacific Ocean: an analysis combining observations with regional modeling" by Jiawei Li et al.
}

Jiawei Li et al.

hzw@mail.iap.ac.cn

Received and published: 21 January 2021

The comment was uploaded in the form of a supplement

Please also note the supplement to this comment:

https://acp.copernicus.org/preprints/acp-2020-1016/acp-2020-1016-AC1- 
Interactive comment 